\title{
Phase separation on the sphere: Patchy particles and self-assembly
}

\author{
M. C. Bott ${ }^{*}$ and J. M. Brader ${ }^{\dagger}$ \\ Soft Matter Theory, University of Fribourg, CH-1700 Fribourg, Switzerland
}

(Received 2 May 2016; published 5 July 2016)

\begin{abstract}
Motivated by observations of heterogeneous domain structure on the surface of cells, we consider a minimal model to describe the dynamics of phase separation on the surface of a spherical particle. Finite-size effects on the curved particle surface lead to the formation of long-lived, metastable states for which the density is distributed in patches over the particle surface. We study the time evolution and stability of these states as a function of both the particle size and the thermodynamic parameters. Finally, by connecting our findings with studies of patchy particles, we consider the implications for self-assembly in many-particle systems.
\end{abstract}

DOI: 10.1103/PhysRevE.94.012603

\section{INTRODUCTION}

Phase separation in bulk systems can proceed via a number of distinct physical mechanisms (such as spinodal decomposition and heterogeneous or homogeneous nucleation), and it is generally well understood. However, the dynamical processes involved are less clear when the system is subject to some form of spatial confinement. This confinement can arise from the presence of external fields, representing, for example, substrates or random obstacles, but it can also be imposed by the geometry of the embedding space. The latter type of confinement is particularly relevant to biological cells, for which the mobile fluid particles constituting the cell membrane are constrained to lie on the surface of a (roughly) spherical body. These membranes exhibit stable domains, the spatial distribution of which is important for many cell properties, e.g., adhesion [1,2]. The composition of these domains and their distribution over the surface of the cell dictates to a large extent the interaction forces between different cells and thus the self-assembly behavior of many-cell systems.

One common view is that the domains on the surface of a cell are a consequence of an arrested or incomplete phase separation, however it remains to be established whether the observed states are permanent or metastable in character. If the heterogeneous domain structure on the cell surface is an equilibrium state, then some stabilizing mechanism is required; the line tension incurred by interfaces between domains would make inhomogeneous phases energetically unfavorable when compared to a fully phase-separated system. Computer simulation studies of simple model systems $[3,4]$ have shown that the size, composition, and dynamics of membrane domains can be regulated by introducing randomly located, immobile objects. These obstacles, embedded within the two-dimensional fluid, serve to hinder macroscopic phase separation and act as a source of quenched disorder. It has been proposed in Refs. [3,4] that the quenched disorder found in real cells, provided by fixed cytoskeletal proteins, could be the key stabilizing mechanism.

An alternative scenario is that the domains are long-lived metastable, rather than equilibrium, states. It is well known from studies of phase separation $[5,6]$ in bulk that quenching

\footnotetext{
*matthiaschristian.bott@unifr.ch

†joseph.brader@unifr.ch
}

the thermodynamic parameters to a state point close to the spinodal will result in very slow phase-separation dynamics. Following the quench, spherical domains of the minority phase form and then slowly merge together, a process known as Ostwald ripening [7]. The ripening process could possibly be slowed down, or even arrested entirely, by the presence of small quantities of an additional species, which sits preferentially at the interfaces between domains.

A requirement for studying domain formation is an understanding of diffusion processes on the sphere. Such studies can be found in the literature in a variety of contexts. The diffusion of noninteracting particles on a spherical surface has been addressed using analytical methods by Ghosh et al. [8]. Marenduzzo and Orlandini have used numerical methods to study diffusive motion on general curved surfaces, and they investigated the coupling between phase separation and local curvature [9]. Fischer and Vink performed many-body simulations on a spherical surface, with the aim to optimize the boundary conditions for simulations of first-order transitions in finite-size systems [10].

Going beyond single-cell properties, assemblies of spherical cells exhibit nontrivial interactions, both with each other and with external substrates. The interaction potential between a pair of cells is strongly influenced by the distribution and size of the domains covering its surface. In this sense, cells may be regarded as a naturally occurring type of "patchy particle," which is the term given to particles with distinct surface sites generating anisotropic interparticle interactions. While synthetically fabricated patchy particles have attractive interaction patches strategically arranged on their surface [11], the domains covering the cell emerge as a result of self-organization. When multiple cells are present in a crowded environment, the influence of competing physical mechanisms, acting both within each cell membrane (line tension, quenched disorder) and between different cells, can generate a complex domain structure.

The phase behavior and equilibrium microstructure of synthetic patchy particles depends upon the number, spatial distribution, and attraction strength of the interaction patches. For example, spherical particles with just two attractive patches will tend to form polymerlike chains, whereas threepatch particles will assemble into open gel-like structures ("empty" liquids) [12]. Recent developments in the controlled fabrication of patchy particles have raised hopes that materials with desired properties may be tailored by prescribing the 
number and geometrical arrangement of the patches $[11,13]$. To understand the collective behavior of natural patchy particles, for which the domains self-organize, it is necessary to understand first the dynamical processes occurring on the surface of individual cells.

In this paper, we investigate how phase separation on the surface of a spherical body can give rise to different domain structures, and then we infer how these domains could influence the self-assembly in systems consisting of many spherical bodies. We do not seek to describe real biological cells, but rather we take these as a motivation for the construction of simple models capturing generic physical features. We will focus first on single-particle properties, investigating how the domains form on the particle surface under various conditions, before proceeding to study how these domains may influence interparticle interactions. In Sec. II we outline the model system to be considered, the theoretical method employed, and the numerical methods used to solve our equations. In Sec. III we investigate the domain formation on a single spherical body, and we infer from this the likely consequences for many-body self-assembly in Sec. IV. Finally, in Sec. V we discuss our findings and provide an outlook.

\section{THEORY}

We will investigate the demixing of a binary fluid on the two-dimensional surface of a large spherical particle. To avoid any confusion with terminology, we will henceforth refer to the large particle as the "mesoparticle" and the smaller, mobile particles constituting the fluid on its surface as the "surface particles." As we are interested in the phenomenology of phase separation and domain formation, we choose for convenience a very simple microscopic model, namely the Gaussian core model (GCM), to represent the surface particles. In the present study, the GCM is employed simply because of its generic demixing properties, rather than as an approximation to any specific physical system. The collective behavior of the GCM on the mesoparticle surface is treated using a well-established mean-field density-functional theory.

\section{A. The Gaussian core model}

To represent the surface particles, we consider a model binary mixture in which the particles interact via the soft repulsive pair potential

$$
\beta v_{i j}(r)=\beta \epsilon_{i j} \exp \left\{-r^{2} / R_{i j}^{2}\right\},
$$

where $\beta=\left(k_{B} T\right)^{-1}$ and the non-negative parameters $\epsilon_{i j}$ and $R_{i j}$ determine the strength and range, respectively, of the interaction between species $i$ and $j$. The GCM was introduced by Stillinger [14] to study phase separation in binary mixtures and has since been studied intensively, both in bulk and at interfaces [15-20]. The model has the advantage that a simple mean-field approximation to the free energy provides good agreement with computer simulation data [21].

When calculating the interaction between surface particles, the separation $r$ entering the pair potential (1) is taken to be the direct, straight-line distance (cutting through the mesosphere), rather than the length of the arc around the surface of the mesosphere.

\section{B. Mean-field free-energy functional}

To describe the collective behavior of the surface particles, we use an approximation to the two-dimensional Helmholtz free-energy functional,

$$
\begin{aligned}
\beta \mathcal{F} & {\left[\left\{\rho_{q}(\mathbf{r}, t)\right\}\right] } \\
= & \sum_{q} \int d \mathbf{r} \rho_{q}(\mathbf{r})\left\{\ln \left[\rho_{q}(\mathbf{r})\right]-1\right\} \\
& +\frac{1}{2} \sum_{q l} \int d \mathbf{r} \int d \mathbf{r}^{\prime} \rho_{q}(\mathbf{r}) \rho_{l}\left(\mathbf{r}^{\prime}\right) \beta v_{q l}\left(\left|\mathbf{r}-\mathbf{r}^{\prime}\right|\right),
\end{aligned}
$$

where the first and second terms provide the ideal and excess (over ideal) contributions, respectively. The subscripts $q$ and $l$ are species labels, and the notation $\left[\left\{\rho_{q}(\mathbf{r}, t)\right\}\right]$ indicates a functional dependence on the one-body density profiles of all species. We set the (physically irrelevant) thermal wavelength equal to unity. For a binary mixture, the species indices are restricted to the values $q, l=1,2$. In bulk, the number density of species $q$ is $\rho_{q}=N_{q} / V$, where $V$ is the area in the twodimensional (2D) case. The total density is $\rho=\rho_{1}+\rho_{2}$.

It is convenient to introduce a concentration variable $x=N_{2} / N$, which enables the species labeled densities to be expressed as $\rho_{1}=(1-x) \rho$ and $\rho_{2}=x \rho$. In these variables, the bulk free energy per particle consists of a sum of two terms, $f \equiv F / N=f_{\text {id }}+f_{\text {ex }}$. The ideal part is given by

$$
\beta f_{\text {id }}=\ln (\rho)-1+(1-x) \ln (1-x)+x \ln (x),
$$

where the contribution $(1-x) \ln (1-x)+x \ln (x)$ is due to the entropy of mixing. The reduced bulk excess free energy per particle is given by

$$
\beta f_{\mathrm{ex}}=\frac{1}{2 \rho}\left[\rho_{1} \rho_{1} \hat{v}_{11}(0)+2 \rho_{1} \rho_{2} \hat{v}_{12}(0)+\rho_{2} \rho_{2} \hat{v}_{22}(0)\right],
$$

where $\hat{v}_{i j}(0)$ is the Fourier transform of the pair potential at zero wave vector $\hat{v}_{i j}(k=0)=\epsilon_{i j}^{*} R_{i j}^{2} \pi$ and $\epsilon_{i j}^{*}=\beta \epsilon_{i j}$.

Expressing $\rho_{1}$ and $\rho_{2}$ in terms of the concentration variable $x$, one obtains

$$
\beta f_{\text {ex }}=\frac{1}{2} \rho\left[(1-x)^{2} \hat{v}_{11}(0)+2 x(1-x) \hat{v}_{12}(0)+x^{2} \hat{v}_{22}(0)\right] .
$$

When the total density $\rho$ becomes sufficiently large, the GCM demixes. To obtain the coexistence curve (binodal) both the chemical potential of each species and the pressure have to be set equal in the coexisting phases (see Appendix A).

\section{Microscopic dynamics of surface particles}

If we assume that the momentum degrees of freedom of the surface particles equilibrate much faster than their positions, then the motion of the surface particles may be modeled using Brownian dynamics [22]. For a multicomponent system, the configurational probability density, $\Psi\left(\left\{\mathbf{r}_{i q}\right\}, t\right)$, describes the probability to find a given particle configuration at time $t$, where $\mathbf{r}_{i q}$ is the coordinate of the $i$ th particle of species $q$. Given an initial state, the time evolution of $\Psi\left(\left\{\mathbf{r}_{i q}\right\}, t\right)$ is given by the Smoluchowski equation [23]

$$
\frac{\partial \Psi\left(\left\{\mathbf{r}_{i q}\right\}, t\right)}{\partial t}=-\sum_{i} \sum_{q} \frac{\partial}{\partial \mathbf{r}_{i q}} \cdot \mathbf{J}_{i q}\left(\left\{\mathbf{r}_{i q}\right\}, t\right),
$$


where the sums are taken over all particles and species. The current of particle $i$ of species $q$ is given by

$$
\begin{aligned}
\mathbf{J}_{i q}\left(\left\{\mathbf{r}_{i q}\right\}, t\right)= & \gamma_{q}^{-1} \Psi\left(\left\{\mathbf{r}_{i q}\right\}, t\right)\left[\mathbf{F}_{i q}\left(\left\{\mathbf{r}_{i q}\right\}, t\right)\right. \\
& \left.-k_{\mathrm{B}} T \frac{\partial}{\partial \mathbf{r}_{i q}} \ln \Psi\left(\left\{\mathbf{r}_{i q}\right\}, t\right)\right],
\end{aligned}
$$

where $\gamma_{q}=k_{\mathrm{B}} T / D_{q}$ is a friction coefficient, $D_{q}$ is the bare diffusion coefficient of species $q$, and $k_{B} T$ is the thermal energy. We will henceforth assume, for simplicity, that all species have equal friction coefficient, $\gamma_{q}=\gamma$. The total force, $\mathbf{F}_{i q}\left(\left\{\mathbf{r}_{i q}\right\}\right)$, is the sum of contributions from interactions and external fields.

\section{Dynamical density-functional theory}

To study phase separation on the surface of a mesoparticle, we will focus on the dynamics of the one-body density of the surface particles. This can be obtained using dynamical density-functional theory (DDFT) [17,24]. Within this approach, the time evolution of the density of species $q$ is given by a generalized diffusion equation

$$
\frac{\partial \rho_{q}(\mathbf{r}, t)}{\partial t}=\frac{\partial}{\partial \mathbf{r}} \cdot\left[\gamma^{-1} \rho_{q}(\mathbf{r}, t) \frac{\partial}{\partial \mathbf{r}} \frac{\delta \mathcal{F}\left[\left\{\rho_{q}(\mathbf{r}, t)\right\}\right]}{\delta \rho_{q}(\mathbf{r}, t)}\right] .
$$

The DDFT equation of motion (8) is obtained from the manybody Smoluchowski equation (6) by (i) integrating over all but one of the particle coordinates, and (ii) approximating the interaction forces using the equilibrium free-energy functional. This second step constitutes an adiabatic assumption. As the adiabatic approximation is well documented, we refer the interested reader to Refs. [17] and [25] for a detailed derivation of Eq. (8).

\section{E. Numerical implementation}

To solve the DDFT equation of motion (8) on the surface of a mesosphere, we must define an appropriate numerical grid. The chosen grid should enable accurate finite-difference schemes for calculating the gradient and divergence of scalarvector fields, as well as an efficient method to compute the convolution of two scalar fields. We find that for the present application, the simplest approach is, in fact, the best choice: we parametrize the sphere using the spherical polar angles $\theta$ and $\phi$. In the following subsections, we report relevant technical details of our numerical solution of (8).

Numerical grid and finite differences: We parametrize the surface of a mesosphere of radius $R$ using the angles $\phi \in$ $[0,2 \pi)$ and $\theta \in[0, \pi]$. The $\phi$ range is divided into $M$ equally spaced points with spacing $d \phi=2 \pi / M$ and the $\theta$ range in $N$ points with spacing $d \theta=\pi / N$. To avoid the singularity at the north $(\theta=0)$ and south $(\theta=\pi)$ poles, we exclude these two points and start our $\theta$ grid at $\theta_{0}=d \theta / 2$ and end it at $\theta_{N-1}=(N-1) d \theta+d \theta / 2=\pi-d \theta / 2$. From Fig. 1, it is evident that the pole regions suffer from oversampling when compared to the area around the equator. However, this disadvantage is compensated for by the ease with which finite differences may be calculated. All fields can be stored in $M$ arrays, and neighboring entries in the array correspond

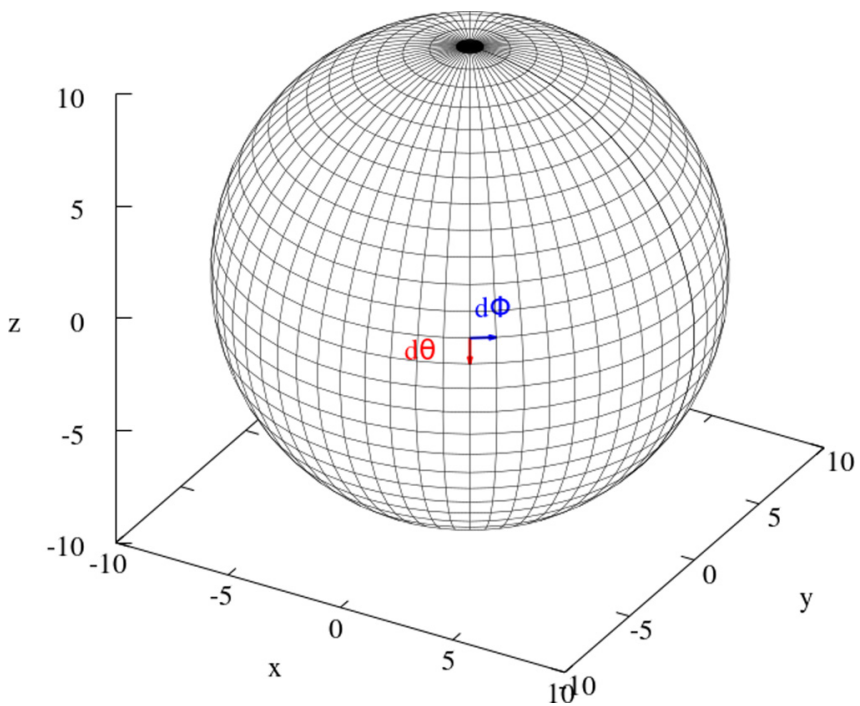

FIG. 1. The numerical grid (here for $M=60$ and $N=30$ ) used to calculate density profiles on the mesoparticle surface. The angular increment $d \phi=2 \pi / M$ points from west to east, and the angular increment $d \theta=p i / N$ points from the north pole to the south pole. This discretization leads to an oversampling of points around the two poles.

to physical neighbors on the sphere. The only complication arises on the edges, $\theta_{0}=d \theta / 2$ and $\theta_{N-1}=\pi-d \theta / 2$. Details of our finite-difference scheme are given in Appendix B.

Convolutions: The nonlocal approximation to the free energy, Eq. (2), generates in Eq. (8) convolution integrals of the form

$$
\int d \Omega^{\prime} f\left(\phi^{\prime}, \theta^{\prime}\right) g\left(\mathbf{r}-\mathbf{r}^{\prime}\right)
$$

where $|\mathbf{r}|=\left|\mathbf{r}^{\prime}\right|=R$ and both $f$ and $g$ are scalar functions. Convolutions on the surface of a unit sphere can be efficiently computed by expanding the scalar fields $f(\phi, \theta)$ and $g(\phi, \theta)$ in spherical harmonics,

$$
f(\phi, \theta)=\sum_{l=0}^{L} \sum_{|m| \leqslant l} a_{l}^{m} Y_{l}^{m}(\phi, \theta) .
$$

In principle, an infinite number of terms are required, but in practice the series may be truncated at a finite value of $L$. It follows from orthogonality, $\int d \Omega \bar{Y}_{l^{\prime}}^{m^{\prime}} Y_{l}^{m}=\delta_{l^{\prime}, l} \delta_{m^{\prime}, m}$, that the coefficients are

$$
a_{l}^{m}=\int d \Omega \bar{Y}_{l}^{m}(\phi, \theta) f(\phi, \theta) .
$$

To compute the convolution (9), we make use of the fact that for two functions $f$ and $g$ defined on the unit sphere, the transform of the convolution is given by a pointwise product of the transforms, namely

$$
(f * g)_{l}^{m}=\sqrt{\frac{4 \pi}{2 l+1}} a_{l}^{m} b_{l}^{0},
$$

where $b_{l}^{0}=\int d \Omega \bar{Y}_{l}^{0}(\phi, \theta) g(\phi, \theta)$. A proof of this statement and further insight into the method can be found in Ref. [26]. Extension of the convolution theorem (12) to spheres of nonunit radius simply requires that Eq. (12) be multiplied by 
a factor $R^{2}$. Spherical harmonic transforms were performed using an open-source $\mathrm{C}$ library $[27,28]$. The computational effort for one transform is of order $(M \times N)^{3 / 2}$.

Time Integration: When solving (8), the spatial grid spacing imposes a bound on the maximum step size $d t$, which can be used to calculate the time evolution. Beyond a critical value of $d t$, the time integration becomes unstable. This is the main drawback of our chosen spatial grid; the local oversampling around the poles leaves very little room to adjust the (global) step size $d t$. It is thus necessary to choose a value of $d t$ sufficiently small that the regions around the poles remain stable. The most reliable method to evolve (8) is simple Euler integration. More sophisticated methods, such as Runge-Kutta integration combined with adaptive step size, do not lead to any significant increase in performance.

\section{RESULTS}

The surface of a mesosphere of radius $R$ represents a finite-size system and thus does not admit a true phase transition. Nevertheless, provided that a sufficient number of surface particles are present, the phase diagram of an infinite planar system offers a useful guide when calculating density dynamics on the mesosphere. The bulk phase diagram of an infinite planar system is shown in Fig. 2 for the parameters $R_{11}=R_{22}=R_{12}=1, \epsilon_{11}^{*}=\epsilon_{22}^{*}=2$, and $\epsilon_{12}^{*}=1.035 \epsilon_{11}^{*}$. State points at which we perform detailed calculations are indicated.

We consider first a mesosphere of radius $R=10 R_{11}$ with a total density of surface fluid particles $\rho R_{11}^{2}=25$ and composition $x=0.5$, corresponding to state point $\mathrm{A}$ in the phase diagram (see Fig. 2). In Fig. 3, we show the density profile of species 1 at four different times. The initial condition is chosen by adding to a constant density several randomly located density peaks and dips of small amplitude.

After a time $t^{*}=t D / R_{11}^{2}=80$, the spinodal instability becomes clearly visible on the scale of the figure. For later times (we show $t^{*}=200$ and 5000), domains form and evolve

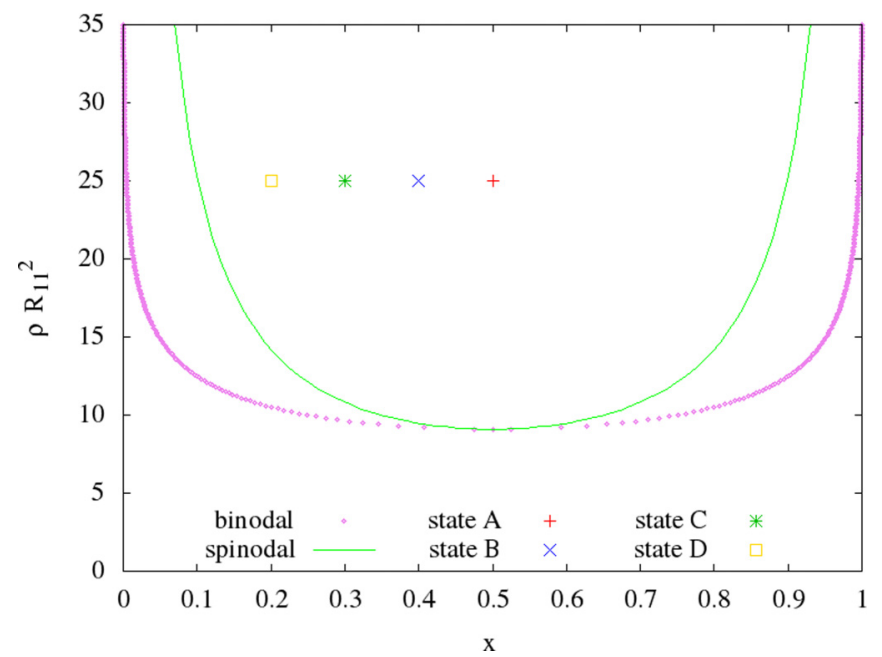

FIG. 2. Phase diagram of the symmetric GCM for the surface particles in an infinite, two-dimensional, planar system. The parameters used are $R_{11}=R_{22}=R_{12}=1, \epsilon_{11}^{*}=\epsilon_{22}^{*}=2$, and $\epsilon_{12}^{*}=1.035 \epsilon_{11}^{*}$. The critical point is located at $\rho R_{11}^{2}=9.094568, x=0.5$.
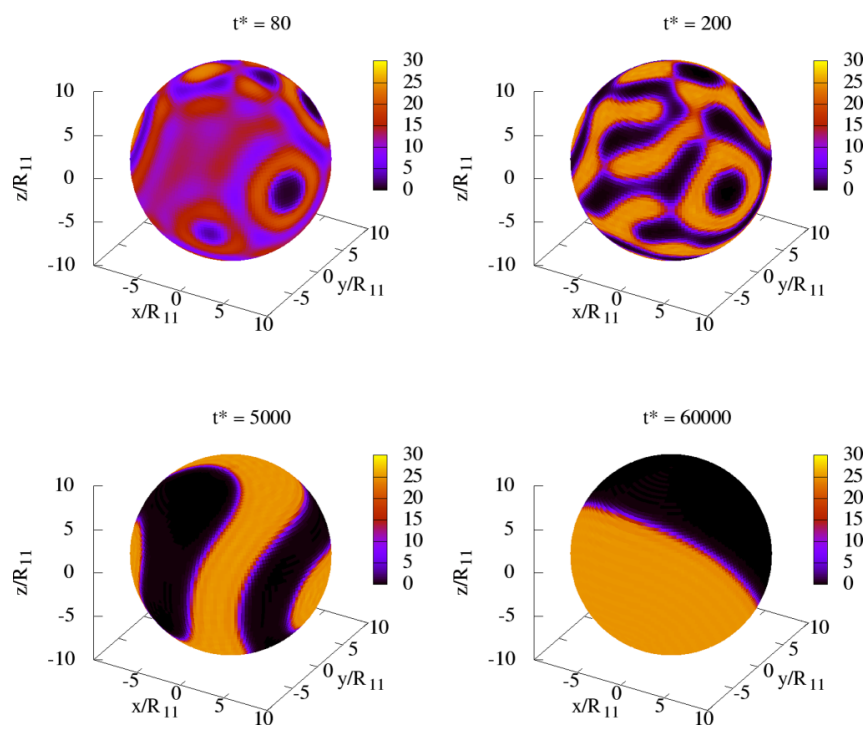

FIG. 3. Larger sphere with $\mathbf{x}=\mathbf{0 . 5}$. The time evolution of the density of species 1 with $\rho R_{11}^{2}=25$, confined on a mesosphere of radius $R=10 R_{11}$. The times shown are $t^{*}=t D / R_{11}^{2}=80,200$, 5000 , and 60000 . The process of spinodal decomposition leads to characteristic density inhomogeneities. In the long-time limit, the line tension is minimized when the interface maps a great circle.

as the system seeks to minimize the length of the boundary between the two phases. At the longest time for which we performed numerical calculations, $t^{*}=60000$, the interfacial region lies on a great circle, which is a consequence of the chosen composition $x=0.5$. We note that the orientation of the final phase-separated state is not correlated with the underlying numerical grid, thus suggesting that our chosen discretization does not introduce any artificial bias into the phase-separation dynamics.

As the DDFT is an adiabatic theory, we can track the time evolution of the free energy. This is shown in Fig. 4 for three different initial conditions where we plot the free energy per

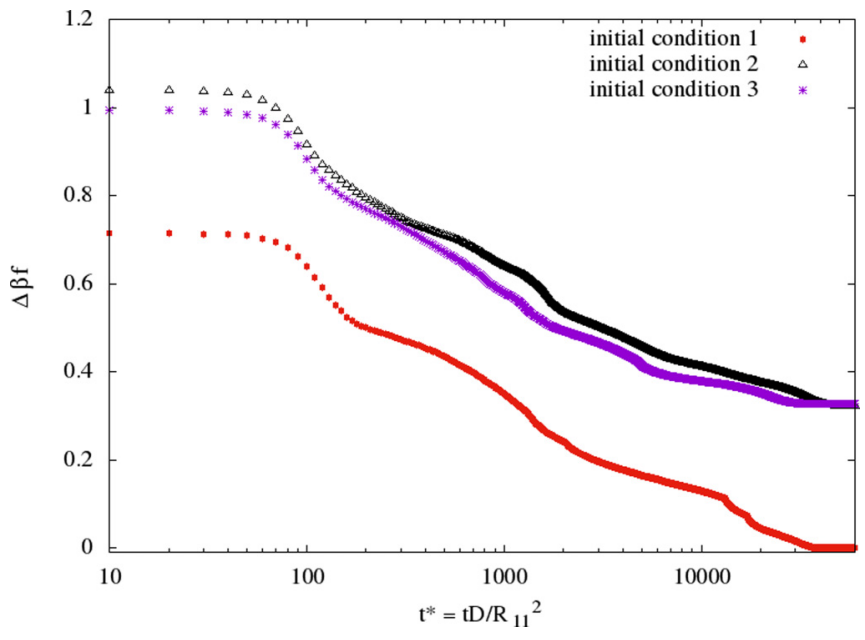

FIG. 4. Larger sphere with $\mathbf{x}=\mathbf{0 . 5}$. Time evolution of the free energy per particle for three different initial conditions of a system with $\rho R_{11}^{2}=25$. Initial condition 1 corresponds to the data shown in Fig. 3. 

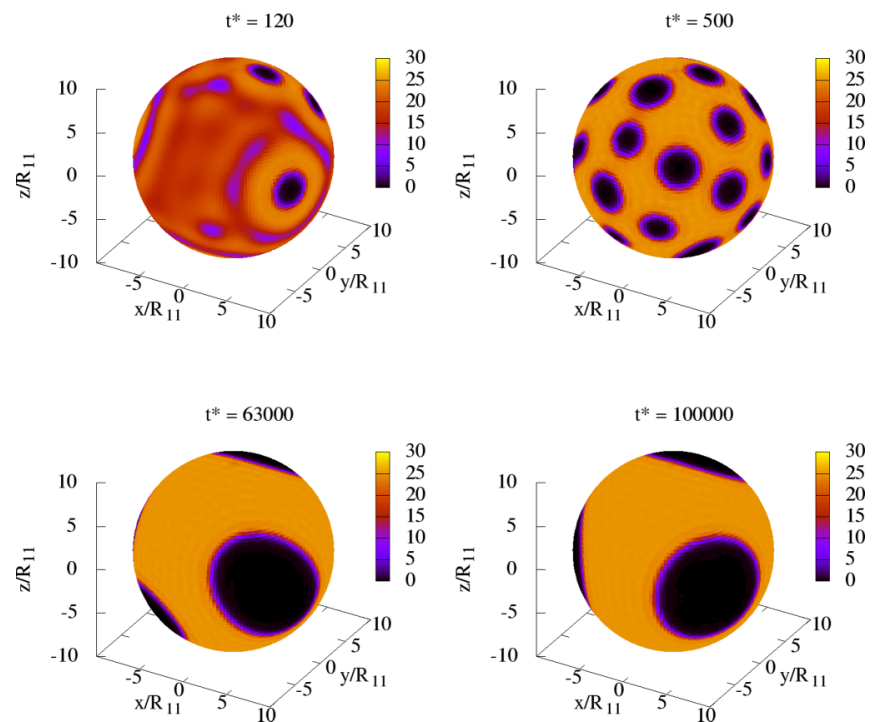

FIG. 5. Larger sphere with $\mathbf{x}=\mathbf{0 . 3}$. Evolution of the density with $\rho R_{11}^{2}=25$ on a mesoparticle with $R=10 R_{11}$. The state at $t^{*}=10^{5}$ is not the final state, but rather a very long-lived metastable state with five patches (two of which are located here around the back of the mesosphere).

particle $\beta f$ minus the long-time value of the free energy. Aside from slight differences arising from different initial conditions, the general behavior of the free-energy relaxation is very similar for all cases investigated; a rapid initial relaxation is followed by a slow decay to equilibrium.

We next consider a composition $x=0.3$, corresponding to state point $\mathrm{C}$ in Fig. 2. The density is shown in Fig. 5 for four different times. In contrast to the behavior for $x=0.5$, the initial stage of the evolution for $x=0.3$ is characterized by the formation of circular islands of the minority phase, which then slowly merge together, a process known as Ostwald ripening [7].

In Fig. 6 we show the corresponding free energy as a function of time. The free energy decreases rapidly whenever

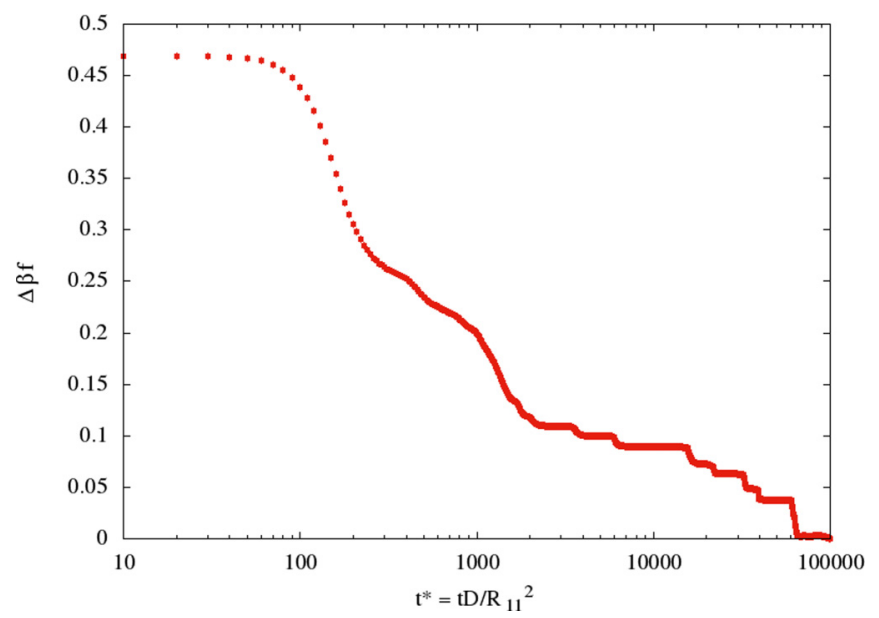

FIG. 6. Larger sphere with $\mathbf{x}=\mathbf{0 . 3}$. Time evolution of the free energy at state point $\rho R_{11}^{2}=25$, corresponding to the density profiles shown in Fig. 5. Sudden decreases in the free energy correspond to events in which two domains merge. This contrasts with the smoother decay observed in Fig. 4.
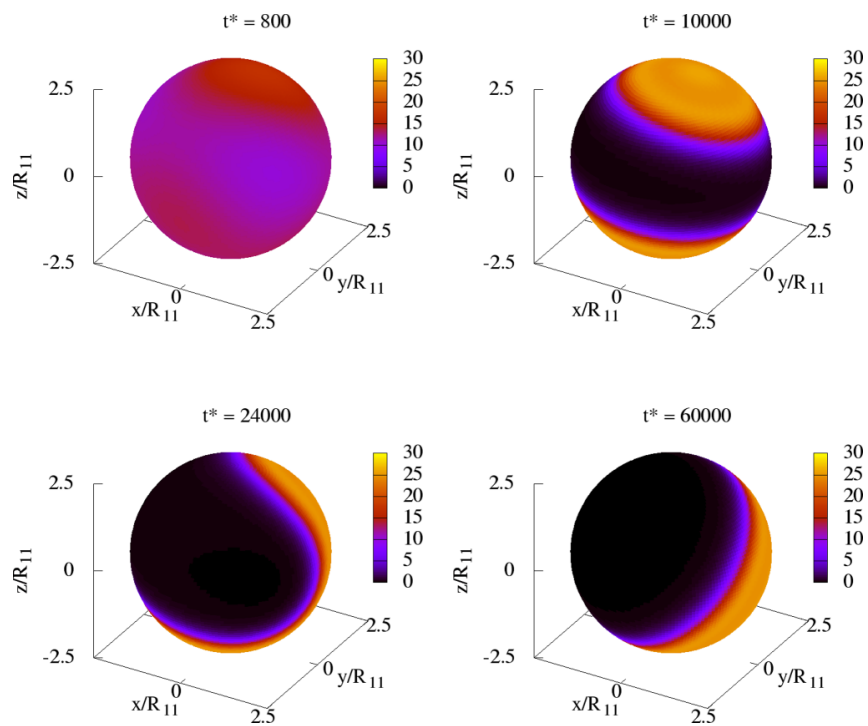

FIG. 7. Smaller sphere with $\mathbf{x}=\mathbf{0 . 5}$. Time evolution of the density with $\rho R_{11}^{2}=25$ on a mesoparticle with radius $R=2.5 R_{11}$. The surface particles become trapped in a "banded" metastable state. For comparison, the density on a larger mesoparticle (see Fig. 3) at the same state point does not display such a banded structure at any point during the time evolution.

two circular patches merge, however these merging events become less frequent as time progresses (note the logarithmic time scale). Even after $t^{*}=10^{5}$, the system has still not attained its final state, but the free energy shows no significant further decrease. The final state shown in Fig. 6 proved to be very stable; the expected completely phase-separated state could not be obtained within the available computation time. For the duration that the surface fluid is trapped in this metastable state, which, according to our calculations, survives many tens of thousands of Brownian time units. During this time window, the mesoparticle could be regarded as a patchy particle, which would surely exhibit anisotropic interactions with neighboring mesoparticles.

We next consider phase separation on a smaller mesoparticle, $R=2.5 R_{11}$, for which finite-size effects become important. A typical example of the time evolution of the density is shown in Fig. 7 and the corresponding free energy in Fig. 8, where we address first the state point $\mathrm{A}$ in the phase diagram ( $\rho=25, x=0.5$ ). When compared with the phase-separation dynamics on the larger mesoparticle (see Fig. 3), we observe from the decay of the free energy that, although the onset time of the initial instability is larger for the smaller sphere, the overall time taken to arrive at the equilibrium state is smaller.

In contrast to the behavior on the larger mesosphere, the density evolves here into a "band" state, where two islands with species 1 form, separated by a band of species 2 particles. This state is stable over a long time, which can be seen in the plateau of the free energy (from $t^{*} \sim 5000$ to $t^{*} \sim 20000$ ), before it finally collapses to reach an equilibrium state qualitatively similar to that found on the larger sphere. The interesting feature here is that, despite the symmetric composition $(x=0.5)$, the time evolution is qualitatively closer to Ostwald ripening than classic spinodal decomposition. This is a finite-size effect, which arises because "long-wavelength modes" (a notion to 


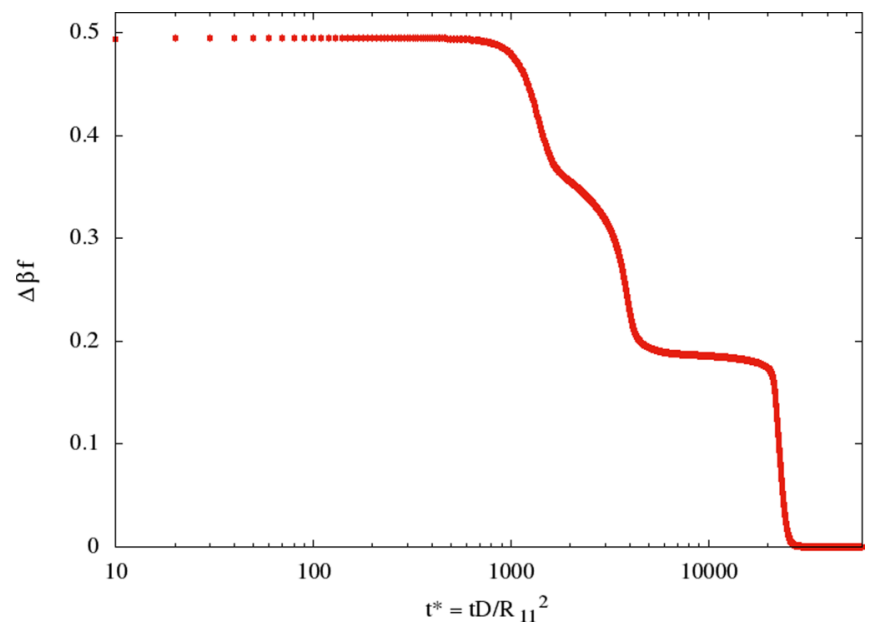

FIG. 8. Smaller sphere with $\mathbf{x}=\mathbf{0 . 5}$. Time evolution of the free energy for state point $\rho R_{11}^{2}=25$ on a mesoparticle with radius $R=2.5 R_{11}$. The sudden decrease in the free energy at around $t^{*}=20000$ corresponds to the breaking of the density "band" around the mesoparticle (see Fig. 7).

be clarified in the following section) are suppressed by the relatively small circumference of the mesosphere, relative to the size of the surface particles.

If the value of $x$ is reduced for fixed $\rho$, the state point moves toward the spinodal and the time taken for the system to reach equilibrium increases. In Fig. 9, we show an example of the density evolution for the value $x=0.3$ (state point $\mathrm{C}$ ). We again observe the formation of a band around the particle, however this metastable state is much longer-lived than that observed for the case $x=0.5$, as can be seen from the time evolution of the free energy shown in Fig. 10. In general, we find that the smaller the value of $x$, the more stable the band structure becomes. In Fig. 10, we show the free energy per particle as a function of time for state points A, B, C, and D in Fig. 2,
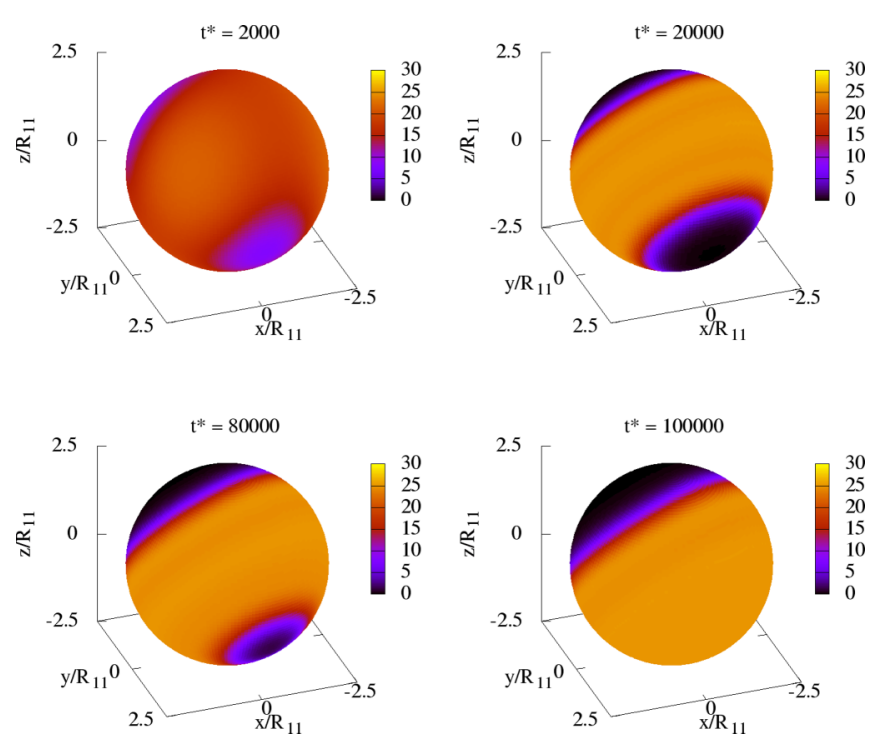

FIG. 9. Smaller sphere with $\mathbf{x}=\mathbf{0 . 3}$. Time evolution of the density with $\rho R_{11}^{2}=25$ on a mesosphere of radius $R=2.5 R_{11}$. The banded state is here more stable than in the case $x=0.5$ (see Fig. 7).

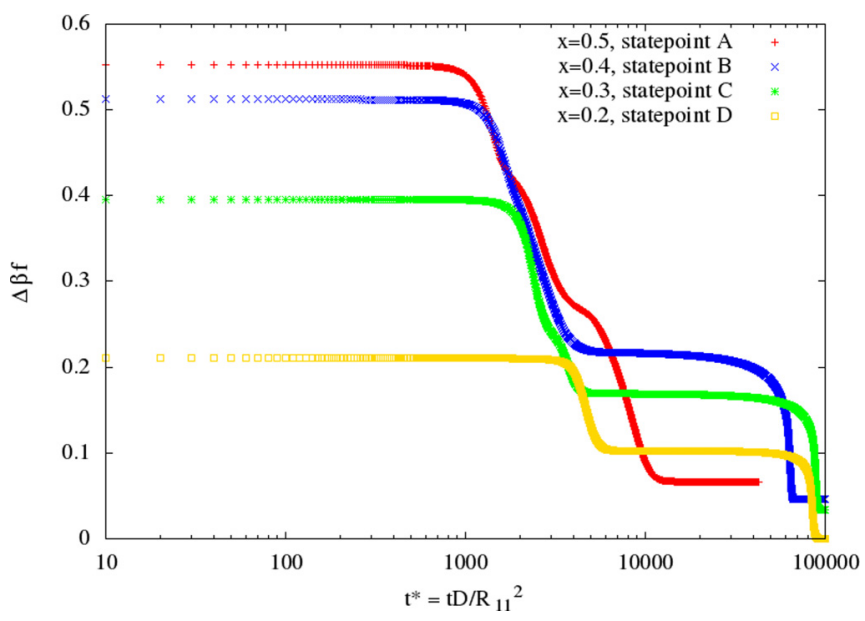

FIG. 10. Smaller sphere with $\mathbf{x}=\mathbf{0 . 2}, \ldots, \mathbf{0 . 5}$. Time evolution of the free energy for $\rho R_{11}^{2}=25$ on a mesosphere of radius $R=2.5 R_{11}$. The plateau in the free energy corresponds to the banded state. The lifetime of the density band increases as $x$ is reduced.

corresponding to $x=0.5,0.4,0.3$, and 0.2 . This enhanced stability of the band structure can be attributed to the fact that the distance between the interfaces increases as the surface coverage of the minority phase is reduced by reducing $x$.

The process of spinodal decomposition in bulk systems is commonly subdivided into different dynamical regimes. In the early stages of phase separation, density gradients are small and the dynamics can be well described using CahnHillard theory (see, e.g., Refs. [29-32]). Early-stage spinodal decomposition is characterized by an exponential growth of low-wavelength density fluctuations [32]. For infinite, flat systems, the fluctuation spectrum is conveniently analyzed using the Fourier transform, which enables unstable wave numbers $k$ to be identified. In the present situation, in which the surface fluid is confined to a spherical surface of finite extent, the analog of the wave number is provided by the $l, m$ labels of the spherical harmonic expansion of the density field.

We define the early stage of spinodal decomposition to be the time window following the quench, for which the linearized theory agrees with a full nonlinear calculation. Deviations indicate the onset of intermediate-stage phase separation. We thus follow [32] and linearize the DDFT equation in the density fluctuation $\tilde{\rho}_{i}(\mathbf{r}, t)=\rho_{i}(\mathbf{r}, t)-\rho_{i}^{b}$. We first express the DDFT equation in the form

$$
\begin{aligned}
\beta \Gamma^{-1} \frac{\partial \tilde{\rho}_{i}(\mathbf{r}, t)}{\partial t}= & \nabla^{2} \tilde{\rho}_{i}(\mathbf{r}, t)-\nabla\left[\tilde{\rho}_{i}(\mathbf{r}, t) \nabla c_{i}^{(1)}(\mathbf{r}, t)\right] \\
& -\rho_{i}^{b} \nabla^{2} c_{i}^{(1)}(\mathbf{r}, t)
\end{aligned}
$$

and we substitute into this expression a functional Taylor expansion of the one-body direct correlation function,

$$
\begin{aligned}
c_{i}^{(1)}(\mathbf{r}, t)= & \left.\sum_{j} c_{i}^{(1)}(\mathbf{r}, t)\right|_{\rho_{j}^{b}} \\
& +\left.\sum_{j} \int d \mathbf{r}^{\prime} c_{i, j}^{(2)}\left(\mathbf{r}, \mathbf{r}^{\prime}, t\right)\right|_{\rho_{j}^{b}} \tilde{\rho}_{j}\left(\mathbf{r}^{\prime}, t\right)+\cdots
\end{aligned}
$$


For the GCM surface fluid, this yields to first order in density fluctuations the following result:

$$
\begin{aligned}
& \beta^{-1} c_{1}^{(1)}(\mathbf{r}, t)=-\rho_{1}^{b} \hat{v}_{11}-\rho_{2}^{b} \hat{v}_{12}-\tilde{\rho}_{1} * v_{11}-\tilde{\rho}_{2} * v_{12}, \\
& \beta^{-1} c_{2}^{(1)}(\mathbf{r}, t)=-\rho_{2}^{b} \hat{v}_{22}-\rho_{1}^{b} \hat{v}_{12}-\tilde{\rho}_{2} * v_{22}-\tilde{\rho}_{1} * v_{12},
\end{aligned}
$$

where the star denotes a convolution. Substitution of (15) into (13) and retaining linear terms yields

$$
\begin{aligned}
& \beta \Gamma^{-1} \frac{\partial \tilde{\rho}_{1}(\mathbf{r}, t)}{\partial t}=\nabla^{2} \tilde{\rho}_{1}(\mathbf{r}, t)-\beta \rho_{1}^{b} \nabla^{2}\left(v_{11} * \rho_{1}+v_{12} * \rho_{2}\right), \\
& \beta \Gamma^{-1} \frac{\partial \tilde{\rho}_{2}(\mathbf{r}, t)}{\partial t}=\nabla^{2} \tilde{\rho}_{2}(\mathbf{r}, t)-\beta \rho_{2}^{b} \nabla^{2}\left(v_{22} * \rho_{2}+v_{12} * \rho_{1}\right) .
\end{aligned}
$$

To identify the regime of early-stage phase separation, we have compared the free energy from the nonlinear DDFT, Eq. (8), with the results obtained by solving Eqs. (16) and (17). Our numerical calculations show where the linearized solution begins to deviate from the full solution of the DDFT equations. For a given mesoparticle radius, we evaluate the density field at a time just prior to this deviation, determine the coefficients in the spherical harmonic expansion, Eq. (10), and then average over the $m$ index. Furthermore, we average over a set of 50 different initial conditions. The resulting averaged coefficient, $\left\langle a_{l}^{m}\right\rangle$, is a function of the index $l$ and indicates which modes of the density field contribute most to the density instability.

In Fig. 11, we show $\left\langle a_{l}^{m}\right\rangle$ as a function of $l$ for different mesoparticle radii. For the familiar case of spinodal decomposition in a flat space, it is standard procedure to analyze the static structure factor in order to identify unstable Fourier modes. In the present situation, in which a liquid is constrained to lie on a finite spherical surface, the $\left\langle a_{l}^{m}\right\rangle$ data shown in Fig. 11 provide an appropriate analog to the structure factor.

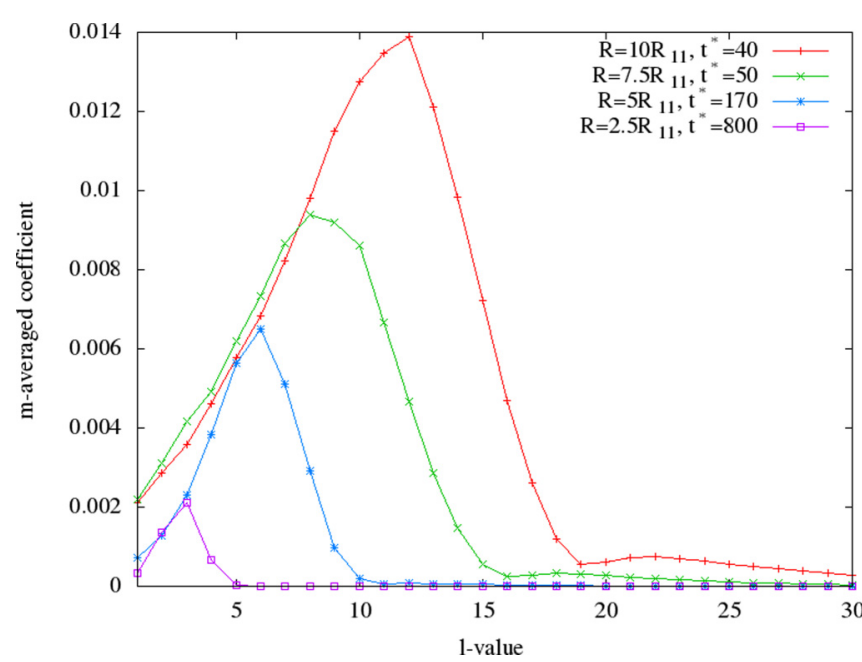

FIG. 11. $l$-modes of the early-stage spinodal decomposition for different mesoparticle sizes. We show the spherical harmonic coefficient for a given $l$-value, averaged over the $m$ indices from $m=-l, \ldots, l$ and averaged over initial conditions. For smaller sphere sizes, the peak shifts to smaller $l$-values.

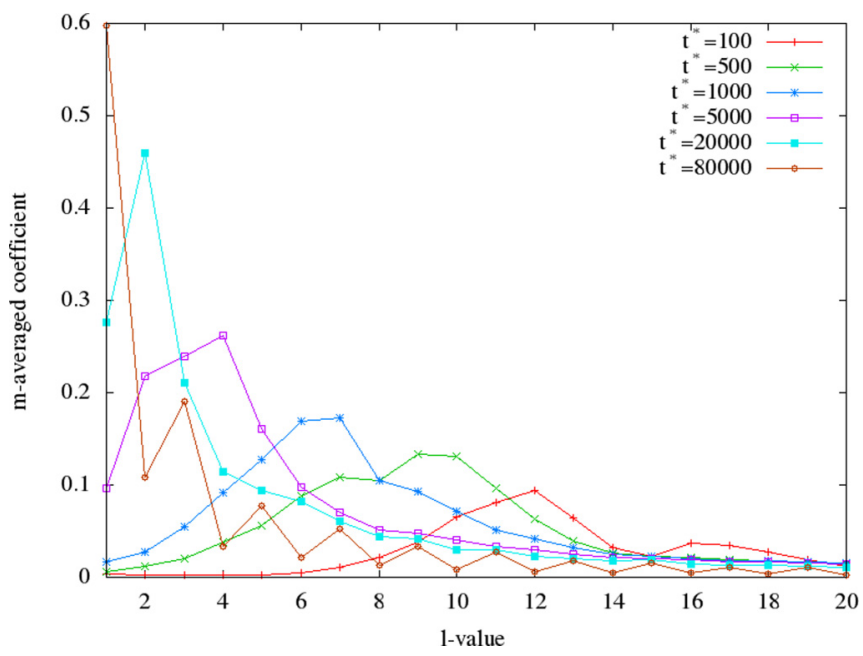

FIG. 12. $l$-modes for different times for a mesoparticle of radius $R=10 R_{11}$. As the density evolves, the smaller $l$-modes give an increasing contribution. In the final state, the dipole dominates.

For smaller sphere sizes, the dominant $l$-values are lower than for larger spheres. An explanation for this effect is that the wavelengths that dominate the instability, the "ripples" on the sphere surface, are independent of the sphere size, and, therefore, on smaller spheres they are described by a smaller $l$-value. Using Jeans' rule, one can identify the wavelength of a spherical harmonic with degree $l$ by $\lambda=2 \pi R /(l+1 / 2)$.

As the density relaxes from its initial to its final state, the $\left\langle a_{l}^{m}\right\rangle$ evolve in time. For a sphere of radius $R=10 R_{11}$ and $x=0.5$, we show in Fig. 12 this time evolution from the end of early-stage spinodal decomposition, all the way to the final state. For times just beyond the early stage of spinodal decomposition, we observe the same behavior as seen in Fig. 11. However, the peak of the curve shifts to smaller values as time increases. In the final state, the surface fluid
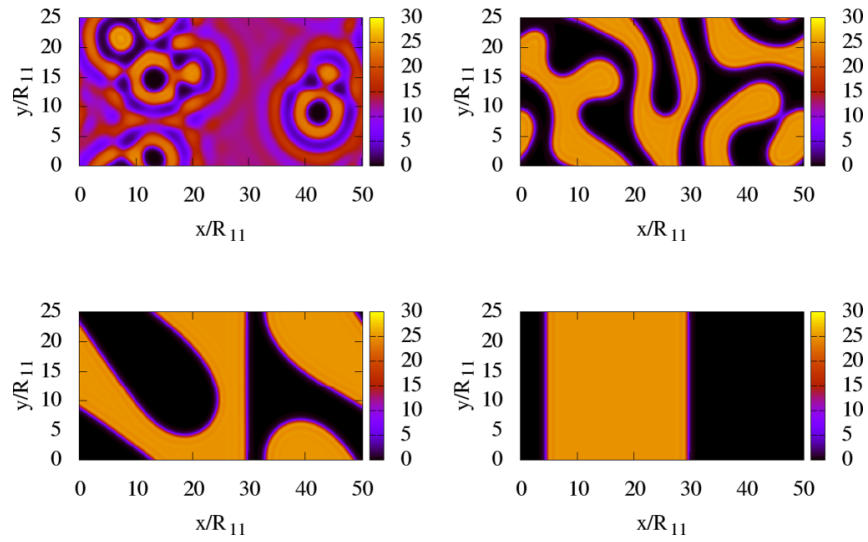

FIG. 13. Time evolution of the density distribution for a Gaussian mixture confined to a flat surface. The system has periodic boundary conditions to ensure the conservation of particles. The number of particles (surface area) is equivalent to the sphere system with radius $R=10 R_{11}$. We see that the dynamics on the flat grid appear to be much faster, since the equilibrium state is reached after roughly $t^{*}=7000$, which took $t^{*}=60000$ on the sphere (see Fig. 3). The corresponding evolution of the free energy can be seen in Fig. 14. 


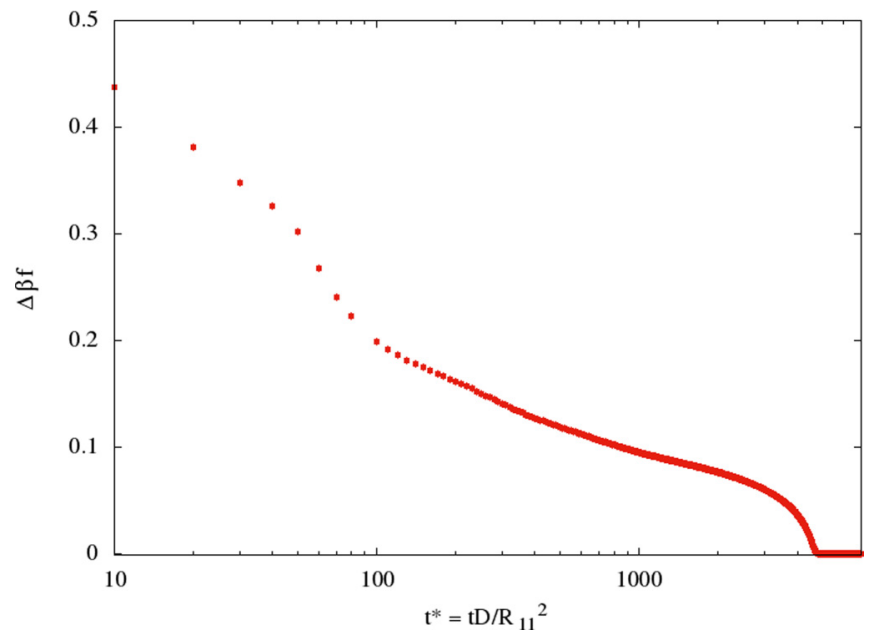

FIG. 14. Free-energy time evolution for the spinodal decomposition on a flat surface with periodic boundary conditions. The flat system shows qualitatively similar behavior to the spherical system. The free energy shows a significant drop in the initial stages of spinodal decomposition and afterward slowly decreases as the system reaches its equilibrium state.

is completely phase-separated and the dominant mode is the dipole $(l=1)$. In all plots, we excluded the $l=0$ contribution, which only represents a homogeneous field and has therefore no contribution to the angular distribution.

The dynamics of phase separation on a mesoparticle can be compared with phase separation in a flat, planar system. For this comparison, we use the same parameters $R_{i j}$ and $\epsilon_{i j}$ as were used previously, and we employ periodic boundary conditions. The number of particles is set equal in the flat and curved systems. The time evolution of the density and the free energy for the flat system are shown in Figs. 13 and 14 , respectively. In both cases, we use $x=0.5$. The main observation is that the dynamics of spinodal decomposition are much faster for the flat system than the corresponding spherical system. The equilibrium state is reached after approximately $t^{*}=7 \times 10^{3}$, compared to the curved system, which took $t^{*}=$ $6 \times 10^{4}$, an order of magnitude longer, to achieve comparable equilibration (see Fig. 3).

From Fig. 13, it is apparent that the periodic boundary conditions artificially constrain the orientation of the interface. This unphysical constraint is absent on the sphere, since its topology does not need any boundary conditions and the interfaces can have arbitrary orientation. From our calculations, it would appear that the finite-size effects associated with smaller mesospheres have a stabilizing effects on the band structure.

\section{INTERACTING MESOPARTICLES}

Going beyond the dynamics of phase separation on a single sphere, we next investigate the interaction between a pair of mesospheres. If two mesospheres are sufficiently close that their surface particles interact, then they exhibit an anisotropic interaction. Understanding the pair interaction can then form a basis for investigating the structures, which may result from self-assembly.
Calculating the interaction potential between two mesoparticles requires as input the distance between two arbitrary points, one located on mesoparticle 1 and the other on mesoparticle 2. For convenience, we fix mesoparticle 1 at the origin of a Cartesian coordinate system (henceforth referred to as the "left particle," with radius $R_{L}$ ). The center of the right particle (radius $R_{R}$ ) is chosen to lie on the positive $x$ axis. The center-to-center distance is $R_{L}+R_{R}+d$, and if $d$ is comparable to the range of the Gaussian interaction $R_{i j}$, then the two particles will influence each other.

The distance $|z|$ between any point $\left(\phi_{L}, \theta_{L}\right)$ on the surface of the left sphere and any point $\left(\phi_{R}, \theta_{R}\right)$ on the surface of the right sphere is given by

$$
\begin{aligned}
|z|^{2}= & \left(R_{L} \sin \theta_{L} \cos \phi_{L}-R_{R} \sin \theta_{R} \cos \phi_{R}-d-R_{L}-R_{R}\right)^{2} \\
& +\left(R_{L} \sin \theta_{L} \sin \phi_{L}-R_{R} \sin \theta_{R} \sin \phi_{R}\right)^{2} \\
& +\left(R_{L} \cos \theta_{L}-R_{R} \cos \theta_{R}\right)^{2} .
\end{aligned}
$$

The external potential exerted on particle species $i=1,2$ on the left sphere by the right sphere is thus given by

$$
\begin{aligned}
\beta V_{\mathrm{ext}}(\theta, \phi)_{L i}= & \beta \int d \Omega^{\prime} \rho_{R 1}\left(\Omega^{\prime}\right) v_{i 1}\left[|z|\left(\Omega, \Omega^{\prime}\right)\right] \\
& +\beta \int d \Omega^{\prime} \rho_{R 2}\left(\Omega^{\prime}\right) v_{i 2}\left[|z|\left(\Omega, \Omega^{\prime}\right)\right] .
\end{aligned}
$$

The external potential acting on the right sphere is then simply obtained by exchanging the labels $R$ and $L$ in the above expression.

In the numerical time integration of the DDFT equation, it is expensive to compute these integrals at each time step. In principle, to solve the time evolution in a fully self-consistent way, the density on each particle surface should be subject at each time step to the instantaneous external field generated by the density distribution on the surface of the other sphere. However, a fully self-consistent solution seems to us to be unnecessary. The two mesoparticles are mobile objects, and, provided the density is not too high, the process of phase separation on each mesoparticle will largely proceed in the absence of significant interaction with the others. From our single mesoparticle studies, we have shown that the patchy domain structure can be a long-lived metastable state. It is thus rather likely that mesoparticles that drift together and interact do so while trapped in a metastable state. More precisely, we assume that the time scale of collisions between mesospheres, $D_{m}^{-1} \rho_{m}^{-2 / 3}$, where $D_{m}$ and $\rho_{m}$ are the diffusion constant and density of the mesospheres, is less than the lifetime of the metastable states on the individual mesosphere surfaces.

Due to the above considerations, we can simplify the problem by considering the interaction of mesospheres with static surface density distributions. These static distributions are obtained from the single-particle calculations presented in Sec. III. For a given interparticle separation, we seek the lowest energy relative orientation of a pair of mesospheres. Using Eq. (18), we can determine for all relative orientations the potential acting upon each mesosphere due to its neighbor and, thus, the dependence of the total free energy on the relative orientation and separation of the mesosphere pair. In Appendix $\mathrm{C}$, we report the techniques required for this calculation. 


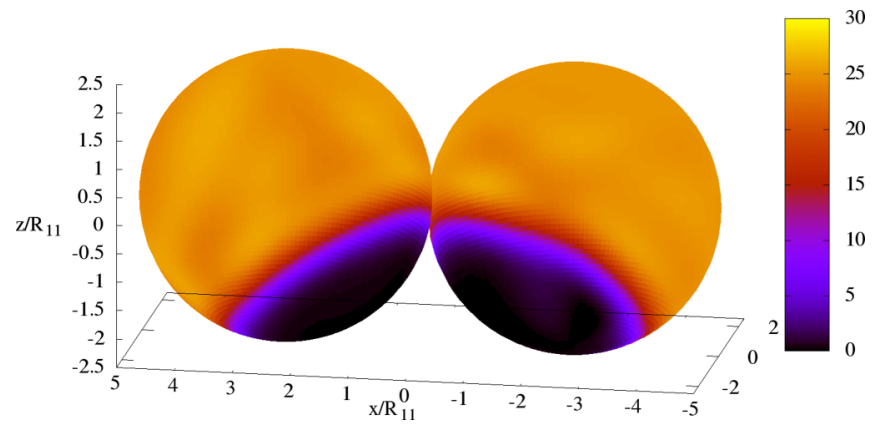

FIG. 15. Two interacting mesospheres with radius $R=2.5 R_{11}$ and $x=0.2$. The configuration shown minimizes the total free energy.

For simplicity, we will limit ourselves to the interaction between mesospheres for which the phase-separation process is fully completed. In Fig. 15, we show the configuration of minimum free energy for $R=2.5 R_{11}$ and $x=0.2$. In this case, the spheres orient such that the interfaces between domains are touching. The choice of mixing parameter $x$ thus specifies the "bond angle" between the two mesospheres. In Fig. 16, we show two different configurations of mesospheres with radius $R=2.5 R_{11}$, but now for $x=0.5$. The first configuration shown (state A) yields the lowest value of the free energy. By flipping one of the spheres (state B), we obtain a state with higher free energy, but which represents a local minimum in

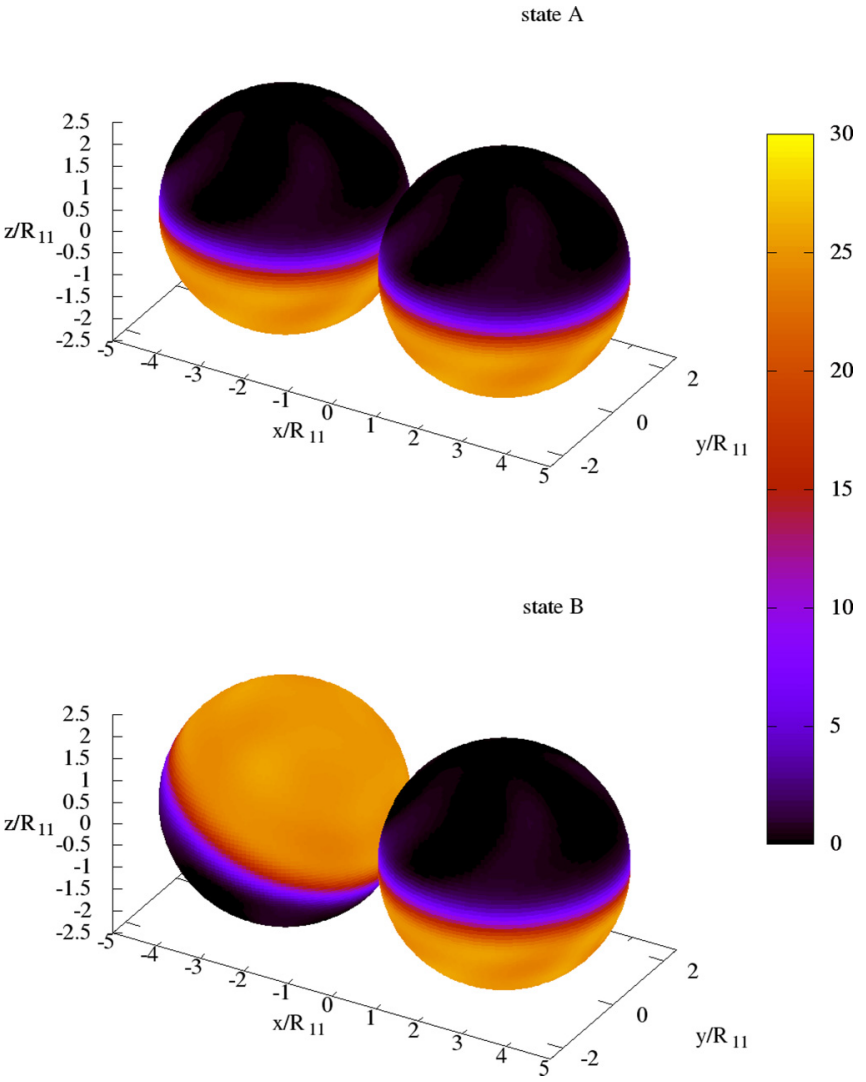

FIG. 16. Two interacting spheres with mixing parameter $x=0.5$. The plot shows two different configurations A and B. State A is the configuration with a minimal energy cost. Turning one of the spheres away from this configuration (state B) leads to an extra energy cost. The energy cost as a function of angle $\theta$ is shown in Fig. 17.

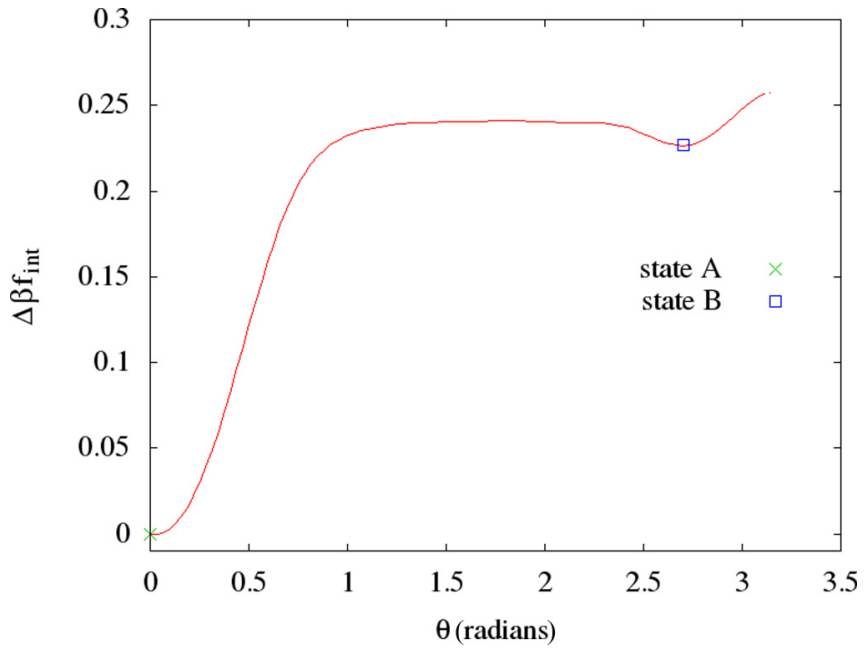

FIG. 17. The energy cost per particle as a function of angle when turning one of the two interacting spheres away from its equilibrium configuration.

the free energy. In Fig. 17, we show the dependence of the free energy on the angle $\theta$ (Euler angle for rotation around the $y$ axis; see also Appendix C).

We would like to emphasize that, for the present GCM surface particles, the interaction forces acting between mesospheres are repulsive. The minimum free-energy configurations identified here correspond to situations of minimal repulsion for a given particle separation. While this is somewhat different from the standard picture of synthetic patchy particles (for which the patches are mutually attractive), we expect the anisotropic repulsion presented by the present model to be important for determining the packing structure of the mesospheres at intermediate and high densities.

The configurations shown in Figs. 15 and 16, together with the free energy in Fig. 17, indicate that fully phase-separated mesospheres will show interesting self-organization behavior, which can be tuned by varying the value of the mixing parameter $x$. For $x=0.5$, it is clear from the minimum energy state A (shown in Fig. 16) that an assembly of many phase-separated mesospheres would build sheets of particles with hexagonal in-plane packing. Indeed, precisely this behavior was found in computer simulations of a closely related model of patchy particles [33]. In this study, the authors considered the self-assembly of hard spheres with discrete attractive patches positioned around the equator. We thus anticipate that our particles with $x=0.5$ will show very similar self-assembly. A distinction between our model and that studied in [33] is that our mesoparticles do not possess an up-down symmetry. Our minimum energy state would have all mesoparticles oriented in the same direction, however the fact that the "flipped state" (state B in Fig. 16) is a local free-energy minimum suggests that a certain fraction of the mesoparticles in the sheet will be flipped with respect to the majority.

For $x \neq 0.5$, the bond angle is no longer zero. In a system of many particles, this would lead in general to a "buckled" sheet of particles that would be subject to geometrical frustration effects. However, for particular choices of $x$ the bond angle can be made compatible with a closed shell of particles. The findings of Ref. [33] support this speculation; simulations were 
performed on systems of hard spheres with a ring of discrete attractive patches lying away from the equator.

\section{CONCLUSIONS}

In this paper, we have studied the process of phase separation on the surface of a sphere using the method of dynamical density-functional theory with a simple mean-field free-energy functional. For larger mesosphere radii, we find standard spinodal decomposition dynamics for an equal mixture, $x=0.5$, leading to a "half-half" final state. As the value of $x$ is reduced toward the spinodel, the phase-separation dynamics are given by the Ostwald ripening scenario, as expected. The long-lived metastable states, consisting of islands of minority phase, could behave as patchy particles with potentially interesting self-assembly properties. An unexpected finding is that smaller mesoparticles do not exhibit typical spinodal decomposition dynamics for any value of $x$. Even for the symmetric mixture with $x=0.5$ the phase separation resembles Ostwald ripening.

For the case of a fully phase-separated larger sphere, we have considered the interaction between pairs of mesoparticles in order to gain insight into possible self-assembly mechanisms. For a pair of mesoparticles in contact with each other, we find the state of minimum free energy to be that where the interfaces between domains are touching (see Figs. 15 and 16) and the mesoparticles have the same orientation. The state for which the particles have opposite orientation is a less favorable metastable minimum of the free energy.

In Ref. [33], the self-assembly of a simplified version of our phase-separated mesoparticles has been studied. Simulations were performed of particles with discrete attractive interaction sites at fixed locations on the particle surface. For particles with an attractive ringlike patch around the equator, self-assembly into particle sheets was identified. When the ring of discrete sites was displaced from the equator, the sheets became bent and frustrated. From our findings, it would appear that a fully phase-separated binary mixture on the surface of each mesoparticle provides an approximate realization of the toy model simulated in Ref. [33]. The self-assembly properties can thus be controlled by varying the mixing parameter $x$ of the surface particles. One can thus speculate about the more complex structures that could arise when mesoparticles in metastable states (e.g., that shown in Fig. 5) interact with each other. We plan to perform extensive Brownian dynamics computer simulations of simplified models to investigate the self-organized structures that can develop in these systems.

Finally, we note that there have been experimental observations on the formation of stripe patterns formed by immiscible ligands coadsorbed on the surface of gold and silver nanoparticles [34]. Supporting atomic simulation studies have shown similar stripe formation for surfactants on spherical surfaces [35]. It would be interesting to see if such structures are captured by the simple density-functional approach employed in the present study.

\section{ACKNOWLEDGMENTS}

This research was supported by the Swiss National Science Foundation through the National Centre of Competence in Research Bio-Inspired Materials.

\section{APPENDIX A}

We recall here the conditions for phase coexistence in the binary mixture [21]. The thermodynamic stability conditions are given by

$$
\begin{array}{r}
\left(\frac{\partial^{2} f}{\partial v^{2}}\right)_{x}>0, \\
\left(\frac{\partial^{2} f}{\partial x^{2}}\right)_{v}>0, \\
\left(\frac{\partial^{2} f}{\partial v^{2}}\right)_{x}\left(\frac{\partial^{2} f}{\partial x^{2}}\right)_{v}-\left(\frac{\partial^{2} f}{\partial v \partial x}\right)^{2}>0,
\end{array}
$$

where $f$ is the Helmholtz free energy per particle and $v=\rho^{-1}$. The first inequality ensures mechanical stability (positive compressibility), the second inequality is the condition against spontaneous demixing at constant volume, and the final inequality ensures stability at constant pressure. With the freeenergy density from Eqs. (3) and (5), the stability conditions can be reduced to

$$
\begin{aligned}
1+\rho \hat{V}_{0}(x) & >0, \\
1-\rho x(1-x) \chi & >0, \\
1+\rho \hat{V}_{1}(x)-\rho^{2} x(1-x) \Delta & >0,
\end{aligned}
$$

where we have defined the following parameters:

$$
\begin{aligned}
\chi & =2 \hat{v}_{12}-\left(\hat{v}_{11}+\hat{v}_{22}\right), \\
\Delta & =\hat{v}_{12}^{2}-\hat{v}_{11} \hat{v}_{22}, \\
\hat{V}_{1}(x) & =(1-x) \hat{v}_{11}+x \hat{v}_{22} .
\end{aligned}
$$

The first inequality (A2a) is always fulfilled for the Gaussian interaction, since $\hat{V}_{0}(x)$ is strictly positive. Phase separation is possible provided that condition (A2b) or condition (A2c) is violated. Below we consider phase coexistence at constant volume, resulting in violation of condition (A2b).

\section{Phase separation at constant volume}

Violation of condition (A2b) requires $\chi>0$,

$$
\chi=\pi\left[2 \epsilon_{12}^{*} R_{12}^{2}-\left(\epsilon_{11}^{*} R_{11}^{2}+\epsilon_{22}^{*} R_{22}^{2}\right)\right]>0 .
$$

Whether phase separation is possible or not depends on the choice of the parameters $\epsilon_{i j}^{*}$ and $R_{i j}$. From Eq. (A3) we see that a simple choice is $R_{11}=R_{22}=R_{12}$ and $\epsilon_{12}^{*}>\epsilon_{11}^{*}=\epsilon_{22}^{*}$. For this choice of parameters, it is physically intuitive that the system might phase-separate, as the energy penalty for unlike particles being close to each other is higher than that for like particles.

The physically unstable region of the phase diagram is given by stability condition (A2b). Instability occurs first, when

$$
1-\rho x(1-x) \chi=0 .
$$

Thus the spinodal line is given by

$$
\rho_{s}(x)=\frac{1}{x(1-x) \chi} .
$$

The binodal (phase coexistence line) is determined by chemical and mechanical equilibrium. This means that the chemical 
potential of both particle species (1 and 2) as well as the pressure is equivalent in both phases $(A$ and $B)$ :

$$
\begin{gathered}
\mu_{1}\left(\rho, x_{A}\right)=\mu_{1}\left(\rho, x_{B}\right), \\
\mu_{2}\left(\rho, x_{A}\right)=\mu_{2}\left(\rho, x_{B}\right), \\
p\left(\rho, x_{A}\right)=p\left(\rho, x_{B}\right) .
\end{gathered}
$$

Chemical potential and pressure are obtained from the freeenergy density via

$$
\begin{aligned}
\mu_{1} & =f-v\left(\frac{\partial f}{\partial v}\right)_{x}-x\left(\frac{\partial f}{\partial x}\right)_{v}, \\
\mu_{2} & =f-v\left(\frac{\partial f}{\partial v}\right)_{x}+(1-x)\left(\frac{\partial f}{\partial x}\right)_{v}, \\
p & =-\left(\frac{\partial f}{\partial v}\right)_{x}
\end{aligned}
$$

After simplification, one finds

$$
\begin{gathered}
\beta \mu_{1}=\ln \left[\rho \lambda^{2}(1-x)\right]+\rho(1-x) \hat{v}_{11}(0)+\rho x \hat{v}_{12}(0), \\
\beta \mu_{2}=\ln \left(\rho \lambda^{2} x\right)+\rho(1-x) \hat{v}_{12}(0)+\rho x \hat{v}_{22}(0), \\
\beta p=\rho+\frac{1}{2} \rho^{2} \hat{V}_{0}(x),
\end{gathered}
$$

with

$$
\hat{V}_{0}(x)=(1-x)^{2} \hat{v}_{11}(0)+2 x(1-x) \hat{v}_{12}(0)+x^{2} \hat{v}_{22}(0) .
$$

\section{Phase separation at constant pressure}

Phase separation at constant pressure is possible provided that condition (A2c) is violated, which is only possible if $\Delta>$ 0 . Using $\hat{v}_{i j}(k=0)=\epsilon_{i j}^{*} R_{i j}^{2} \pi$, we obtain

$$
\Delta=\pi^{2}\left[\left(\epsilon_{12}^{*}\right)^{2} R_{12}^{4}-\epsilon_{11}^{*} \epsilon_{22}^{*} R_{11}^{2} R_{22}^{2}\right]>0 .
$$

The unstable region of the phase diagram is also given by condition (A2c), and we obtain the spinodal line from

$$
1+\rho \hat{V}_{1}(x)-\rho^{2} x(1-x) \Delta=0 .
$$

Solving for the density leads to

$$
\rho_{s}(x)=\frac{\hat{V}_{1}(x)+\sqrt{\hat{V}_{1}(x)^{2}+4 x(1-x) \Delta}}{2 x(1-x) \Delta} .
$$

To determine the binodal line, it is convenient to work with the Gibbs free-energy density $g(x, p)$, where the pressure $p$ is the independent variable. Thus we have to perform a Legendre transform of the free-energy density $f(x, v)$,

$$
g(x, p)=f(x, v(p, x))+p v(p, x)=f(x, \rho(p, x))+\frac{p}{\rho(p, x)} .
$$

Therefore, we need the density $\rho$ as a function of pressure, which is obtained by inverting Eq. (A8). The quadratic equation in $\rho$ has two solutions: one is negative and therefore physically irrelevant, and the other one is given by

$$
\rho(p, x)=\frac{-1+\sqrt{1+2 \beta p \hat{V}_{0}(x)}}{\hat{V}_{0}(x)} .
$$

Finally, the Gibbs free-energy density is

$$
\begin{aligned}
\beta g(x, p)= & \ln \left[\lambda^{2} \rho(p, x)\right]-1+(1-x) \ln (1-x)+x \ln (x) \\
& +\frac{1}{2} \rho(p, x) \hat{V}_{0}(x)+\frac{\beta p}{\rho(p, x)} .
\end{aligned}
$$

With this thermodynamic potential, the coexistence condition is given by

$$
\left.\left(\frac{\partial g}{\partial x}\right)_{p}\right|_{x_{A}}=\left.\left(\frac{\partial g}{\partial x}\right)_{p}\right|_{x_{B}}=\frac{g\left(x_{A}, p\right)-g\left(x_{B}, p\right)}{x_{A}-x_{B}} .
$$

Above a critical pressure $p_{\text {crit }}$, the derivative of the Gibbs free energy shows the typical loops. For fixed $p$, Eq. (A11) can be solved numerically using the common tangent construction, which will not be presented here. Resulting phase diagrams for various parameters of $R_{i j}$ and $\epsilon_{i j}^{*}$ can be found in Ref. [15].

\section{APPENDIX B}

The gradient of a scalar field $f(\phi, \theta)$ defined on the surface of a sphere with radius $R$ is given by

$$
\nabla f(\phi, \theta)=\frac{1}{R^{2} \sin \theta} \frac{\partial f}{\partial \phi} \mathbf{e}_{\phi}+\frac{1}{R} \frac{\partial f}{\partial \theta} \mathbf{e}_{\theta} .
$$

To numerically compute the gradient, we use a central difference scheme for the $\phi$ component,

$$
(\nabla f)_{\phi}\left(\phi_{i}, \theta_{j}\right)=\frac{f\left(\phi_{i+1}, \theta_{j}\right)-f\left(\phi_{i-1}, \theta_{j}\right)}{2 R^{2} \sin \theta d \phi},
$$

where $i=1, \ldots, M-2$. We can easily extend the scheme over the edges of the numerical grid by using $\left(\phi_{0}\right.$ and $\left.\phi_{M-2}\right)$ and $\left(\phi_{1}\right.$ and $\left.\phi_{M-1}\right)$ to obtain the gradient at the position $\left(\phi_{M-1}, \theta_{j}\right)$ and at $\left(\phi_{0}, \theta_{j}\right)$. Similarly the $\theta$ component is computed via

$$
(\nabla f)_{\theta}\left(\phi_{i}, \theta_{j}\right)=\frac{f\left(\phi_{i}, \theta_{j+1}\right)-f\left(\phi_{i}, \theta_{j-1}\right)}{2 R d \theta},
$$

where $j=1, \ldots, N-2$. When computing this component of the gradient on the edges of the numerical grid, one has to keep in mind that the $j=0$ row of the array bends around the north pole (also, the $j=N-1$ row bends around the south pole). The gradient of the points surrounding the north pole is thus given by

$$
(\nabla f)_{\theta}\left(\phi_{i}, \theta_{0}\right)=\frac{f\left(\phi_{i}, \theta_{1}\right)-f\left(\phi_{i+M / 2}, \theta_{0}\right)}{2 R d \theta},
$$

where $i=0, \ldots, M / 2-1$, and

$$
(\nabla f)_{\theta}\left(\phi_{i}, \theta_{0}\right)=\frac{f\left(\phi_{i}, \theta_{1}\right)-f\left(\phi_{i-M / 2}, \theta_{0}\right)}{2 R d \theta},
$$

where $i=M / 2, \ldots, M-1$. We compute the gradient's $\theta$ component on points surrounding the south pole by using $\theta_{N-1}$ and $\theta_{N-2}$ on the right-hand side.

The divergence of a vector field $\mathbf{A}(\phi, \theta)$ defined on the surface of a sphere is given by

$$
\boldsymbol{\nabla} \cdot \mathbf{A}(\phi, \theta)=\frac{1}{R \sin \theta} \frac{\partial}{\partial \phi} A_{\phi}+\frac{1}{R \sin \theta} \frac{\partial}{\partial \theta}\left(A_{\theta} \sin \theta\right) .
$$

The finite-difference method described above for the gradient can again be employed. However, when computing the second term on the right-hand side of (B6), it must be recalled that 
the $\theta$ component of a vector field on the sphere points in the direction of the south pole. On the edges $\left(\theta_{0}\right.$ and $\left.\theta_{N-1}\right)$, this leads to a sign change in the finite-difference scheme. For points around the north pole, the second term of Eq. (B6) is given by

$$
\begin{aligned}
\frac{\partial}{\partial \theta} & {\left[A_{\theta}\left(\phi_{i}, \theta_{0}\right) \sin \theta_{0}\right] } \\
& =\frac{A_{\theta}\left(\phi_{i}, \theta_{1}\right) \sin \theta_{1}+A_{\theta}\left(\phi_{i+M / 2}, \theta_{0}\right) \sin \theta_{0}}{2 d \theta},
\end{aligned}
$$

where $i=0, \ldots, M / 2-1$, and

$$
\begin{aligned}
\frac{\partial}{\partial \theta} & {\left[A_{\theta}\left(\phi_{i}, \theta_{0}\right) \sin \theta_{0}\right] } \\
& =\frac{A_{\theta}\left(\phi_{i}, \theta_{1}\right) \sin \theta_{1}+A_{\theta}\left(\phi_{i-M / 2}, \theta_{0}\right) \sin \theta_{0}}{2 d \theta},
\end{aligned}
$$

where $i=M / 2, \ldots, M-1$. For the points surrounding the south pole,

$$
\begin{aligned}
\frac{\partial}{\partial \theta}\left[A_{\theta}\left(\phi_{i}, \theta_{N-1}\right) \sin \theta_{0}\right] & (\mathrm{B} 11) \\
= & \frac{-A_{\theta}\left(\phi_{i+M / 2}, \theta_{N-1}\right) \sin \theta_{N-1}-A_{\theta}\left(\phi_{i}, \theta_{N-2}\right) \sin \theta_{N-2}}{2 d \theta},
\end{aligned}
$$

where $i=0, \ldots, M / 2-1$, and

$$
\begin{aligned}
\frac{\partial}{\partial \theta} & {\left[A_{\theta}\left(\phi_{i}, \theta_{N-1}\right) \sin \theta_{0}\right] } \\
& =\frac{-A_{\theta}\left(\phi_{i+M / 2}, \theta_{N-1}\right) \sin \theta_{N-1}-A_{\theta}\left(\phi_{i}, \theta_{N-2}\right) \sin \theta_{N-2}}{2 d \theta},
\end{aligned}
$$

where $i=M / 2, \ldots, M-1$.

\section{APPENDIX C}

To compute the interaction energy between two mesospheres in various configurations, the density field on the sphere needs to be rotated. Because of the nonuniform spherical grid, this is a nontrivial task. For rotations in the $\phi$ direction, one can simply map each point onto the neighboring point. Unfortunately, for rotations in the direction of $\theta$ this is not possible (see also Fig. 1). Fortunately, we can work around this problem by performing the rotations in the space of spherical harmonic functions. One can compute rotation matrices, which act upon the coefficients of the spherical harmonic expansion, and hence the rotation is done independently of the numerical grid. In this appendix, we show how to compute these rotation matrices.

An arbitrary rotation of a rigid body can be specified using the three Euler angles $\alpha, \beta$, and $\gamma$. In a Cartesian coordinate system, this rotation is generally defined as a rotation around the $z$ axis by angle $\alpha$, followed by a rotation around the new $y$ axis with angle $\beta$, and finally a rotation around the new $z$ axis with angle $\gamma$. In our spherically symmetric case, any orientation of the density field can be achieved by using only angles $\alpha \in[0,2 \pi)$ and $\beta \in[0, \pi]$. The rotated expansion coefficients $\hat{a}_{l}^{m^{\prime}}$ can be expressed using the following rotation matrix:

$$
\begin{aligned}
\hat{a}_{l}^{m^{\prime}} & =\sum_{m=-l}^{l} T_{l}^{m^{\prime}, m}(\alpha, \beta, \gamma) a_{l}^{m}, \\
T_{l}^{m^{\prime}, m} & =e^{-i m \gamma} H_{l}^{m^{\prime}, m}(\beta) e^{i m \alpha} .
\end{aligned}
$$

We see that the rotation in the $\phi$ direction around the $x$ axis by angle $\alpha$ and $\beta$ is achieved by a simple multiplication with an exponential (the rotation matrix is diagonal). Rotation in the $\theta$ direction by angle $\beta$ is given through the matrix $H_{l}^{m^{\prime}, m}(\beta)$, which becomes larger as one goes to higher $l$-subspaces. Here we show how to compute this matrix using recursion, slightly modified from that described in Ref. [36].

Step 1. We compute all $H_{l}^{0, m}(\beta)$ for $m=0, \ldots, l$ for every subspace $l$ up to $L+1$, where $L$ is the desired upper limit. These coefficients are given by the associated Legendre polynomials $P_{l}^{m}(x)$,

$$
H_{l}^{0, m}=(-1)^{m} \sqrt{\frac{(n-|m|) !}{(n+|m|) !}} P_{l}^{|m|}[\cos (\beta)] .
$$

Using the symmetry rule

$$
H_{l}^{m^{\prime}, m}(\beta)=H_{l}^{-m^{\prime},-m}(\beta),
$$

we also obtain $H_{l}^{0, m}$ for $m=-1, \ldots,-l$. Furthermore, we use a second symmetry relation to get $H_{l}^{m^{\prime}, 0}(\beta)$ for $-l \leqslant m^{\prime} \leqslant$ $l$,

$$
H_{l}^{m^{\prime}, m}(\beta)=H_{l}^{m, m^{\prime}}(\beta) .
$$

Step 2. In every subspace $l$, we compute $H_{l}^{1, m}(\beta)$ for $m=$ $1, \ldots, l$ using the following recursion:

$$
\begin{aligned}
H_{l}^{1, m}(\beta)= & \frac{1}{b_{l+1}^{0}}\left\{b_{l+1}^{-m-1} \frac{1-\cos (\beta)}{2} H_{l+1}^{0, m+1}\right. \\
& \left.-b_{l+1}^{m-1} \frac{1+\cos (\beta)}{2} H_{l+1}^{0, m-1}-a_{l}^{m} \sin (\beta) H_{l+1}^{0, m}\right\},
\end{aligned}
$$

with

$$
a_{l}^{m}=\sqrt{\frac{(l+1+m)(l+1-m)}{(2 l+1)(2 l+3)}}
$$

and

$$
\begin{aligned}
b_{l}^{m} & =\operatorname{sgn}(m) \sqrt{\frac{(l-m-1)(l-m)}{(2 l+1)(2 l+1)},} \\
\operatorname{sgn}(m) & = \begin{cases}1, & m \geqslant 0, \\
-1, & m<0 .\end{cases}
\end{aligned}
$$

Using the symmetry relations $(\mathrm{C} 1)$ and $(\mathrm{C} 2)$, we also obtain $H_{l}^{-1, m}(\beta)$ for $m=-1, \ldots,-l, H_{l}^{m^{\prime}, 1}(\beta)$ for $m^{\prime}=0, \ldots, l$, and $H_{l}^{m^{\prime},-1}(\beta)$ for $m^{\prime}=-1, \ldots,-l$.

Step 3. We compute $H_{l}^{m^{\prime}+1, m}(\beta)$ for $m^{\prime}=1, \ldots, l-1$ and $m=m^{\prime}, \ldots, l$ within every subspace $l$ using

$$
\begin{aligned}
H_{l}^{m^{\prime}+1, m}(\beta)= & \frac{1}{d_{l}^{m^{\prime}}}\left\{d_{l}^{m^{\prime}-1} H_{l}^{m^{\prime}-1, m}(\beta)\right. \\
& \left.-d_{l}^{m-1} H_{l}^{m^{\prime}, m-1}(\beta)+d_{l}^{m} H_{l}^{m^{\prime}, m+1}(\beta)\right\},
\end{aligned}
$$


where

$$
d_{l}^{m}=\frac{\operatorname{sgn}(m)}{2}[(l-m)(l+m+1)]^{1 / 2} .
$$

With symmetry relation (C2), we can complete all missing entries in the positive $m, m^{\prime}$ triangle in each subspace $l$. The negative $m, m^{\prime}$ triangle is given through symmetry rule (C1).

Step 4 . We compute $H_{l}^{-1, m}(\beta)$ in every subspace $l$ for $m=$ $1, \ldots, l$,

$$
\begin{aligned}
H_{l}^{-1, m}(\beta)= & \frac{1}{b_{l+1}^{0}}\left\{b_{l+1}^{m+1} \frac{1-\cos (\beta)}{2} H_{l+1}^{0,-m-1}(\beta)\right. \\
& -b_{l+1}^{-m+1} \frac{1+\cos (\beta)}{2} H_{l+1}^{0,-m+1}(\beta) \\
& \left.-a_{l}^{-m} \sin (\beta) H_{l+1}^{0,-m}(\beta)\right\} .
\end{aligned}
$$

Again using symmetry relation (C1), we can add the obtained values to the positive $m^{\prime}$, negative $m$ triangle.

Step 5. Finally, we compute the coefficients $H_{l}^{m^{\prime}-1, m}(\beta)$ for $m^{\prime}=-1, \ldots,-l+1$ and $m=-m^{\prime}, \ldots, l$,

$$
\begin{aligned}
H_{l}^{m^{\prime}-1, m}(\beta)= & \frac{1}{d_{l}^{m^{\prime}-1}}\left\{d_{l}^{m^{\prime}} H_{l}^{m^{\prime}+1, m}(\beta)\right. \\
& \left.+d_{l}^{m-1} H_{l}^{m^{\prime}, m-1}(\beta)-d_{l}^{m} H_{l}^{m^{\prime}, m+1}\right\},
\end{aligned}
$$

and we complete the missing entries for the negative $m^{\prime}$, positive $m$ triangle using symmetry rule (C2). The matrix entries of this triangle can then be projected onto the positive $m^{\prime}$, negative $m$ triangle with symmetry relation $(\mathrm{C} 1)$, which leaves us with the completed rotation matrix.
[1] P.-F. Lenne and A. Nicolas, Soft Matter 5, 2841 (2009).

[2] D. Lingwood and K. Simons, Science 327, 46 (2010).

[3] R. L. C. Vink, Soft Matter 5, 4388 (2009).

[4] T. Fischer and R. L. C. Vink, J. Chem. Phys. 134, 055106 (2011).

[5] W. Li and J. C. Lee, Physica A 202, 165 (1994).

[6] J. C. Lee, Physica A 210, 127 (1994).

[7] A. Onuki, Phase Transition Dynamics (Cambridge University Press, Cambridge, 2007).

[8] A. Ghosh, J. Samuel, and S. Sinha, Europhys. Lett. 98, 30003 (2012).

[9] D. Marenduzzo and E. Orlandini, Soft Matter 9, 1178 (2013).

[10] T. Fischer and R. L. C. Vink, J. Phys.: Condens. Matter 22, 104123 (2010).

[11] E. Bianchi, R. Blaak, and C. N. Likos, Phys. Chem. Chem. Phys. 13, 6397 (2011).

[12] E. Bianchi, J. Largo, P. Tartaglia, E. Zaccarelli, and F. Sciortino, Phys. Rev. Lett. 97, 168301 (2006).

[13] S. C. Glotzer and M. J. Solomon, Nat. Mater. 6, 557 (2007).

[14] F. H. Stillinger, J. Chem. Phys. 65, 3968 (1976).

[15] A. J. Archer and R. Evans, Phys. Rev. E 64, 041501 (2001).

[16] A. J. Archer and R. Evans, J. Phys.: Condens. Matter 14, 1131 (2002).

[17] A. J. Archer and R. Evans, J. Chem. Phys. 118, 9726 (2003).

[18] A. J. Archer, C. N. Likos, and R. Evans, J. Phys.: Condens. Matter 16, L297 (2004).

[19] A. J. Archer, R. Evans, R. Roth, and M. Oettel, J. Chem. Phys. 122, 084513 (2005).
[20] A. J. Archer, M. Schmidt, and R. Evans, Phys. Rev. E 73, 011506 (2006).

[21] A. A. Louis, P. G. Bolhuis, and J. P. Hansen, Phys. Rev. E 62, 7961 (2000).

[22] C. W. Gardiner, Handbook of Stochastic Methods (Springer, Berlin, 1985).

[23] J. K. G. Dhont, An Introduction to Dynamics of Colloids (Elsevier, Amsterdam, 1996).

[24] U. M. B. Marconi and P. Tarazona, J. Chem. Phys. 110, 8032 (1999).

[25] J. Reinhardt and J. M. Brader, Phys. Rev. E 85, 011404 (2012).

[26] J. R. Driscoll and D. M. Healy, Adv. Appl. Math. 15, 202 (1994).

[27] Christopher Cantalupo, ccSHT, http://crd-legacy.lbl.gov/ cmc/ ccSHTlib/doc/

[28] M. Frigo and S. G. Johnson, Proc. IEEE 93, 216 (2005).

[29] F. F. Abraham, J. Chem. Phys. 64, 2660 (1976).

[30] J. K. G. Dhont, J. Chem. Phys. 105, 5112 (1996).

[31] R. Evans and M. M. Telo da Gama, Mol. Phys. 38, 687 (1979).

[32] A. J. Archer and R. Evans, J. Chem. Phys. 121, 4246 (2004).

[33] Z. Zhang and S. C. Glotzer, Nano Lett. 4, 1407 (2004).

[34] A. M. Jackson, J. W. Myerson, and F. Stellacci, Nat. Mater. 3, 330 (2004).

[35] C. Singh, P. K. Ghorai, M. A. Horsch, A. M. Jackson, R. G. Larson, F. Stellacci, and S. C. Glotzer, Phys. Rev. Lett. 99, 226106 (2007)

[36] N. A. Gumerov and R. Duraiswami, arXiv:1403.7698. 